\title{
Evaluación de biomarcadores de estrés oxidativo en diferentes poblaciones de adultos de Cydia pomonella de la Patagonia argentina
}

Maero, E. y Anguiano, O. L.

\begin{abstract}
RESUMEN
En el ambiente natural, los organismos están expuestos a factores ambientales prooxidantes. En este trabajo se evaluaron biomarcadores antioxidantes en insectos adultos de Cydia pomonella de diferentes poblaciones de campo y de una cepa de laboratorio. Las poblaciones de campo provinieron de las provincias de Neuquén (Centenario), Río Negro (Allen, General Roca 1, General Roca 2, General Roca 3 y General Godoy) y Chubut (Sarmiento 1 y Sarmiento 2). Se determinaron antioxidantes enzimáticos -como catalasa (CAT), superóxido dismutasa (SOD) y glutatión S-transferasa (GST)- y no enzimáticos, nivel de glutatión (GSH) endógeno. Las actividades GST determinadas en las polillas provenientes de las poblaciones de campo resultaron significativamente menores a las medidas en la cepa de laboratorio. La actividad CAT detectada en los individuos provenientes de General Roca 1 resultó 2,13 veces menor a la de las polillas de laboratorio. No se observaron diferencias significativas entre poblaciones en los valores determinados de actividad SOD. El nivel de GSH determinado en las polillas de Sarmiento 2 resultó significativamente diferente y fue 1,57 veces mayor al de los individuos de laboratorio. Con excepción de las polillas provenientes de General Godoy, el resto de las poblaciones de campo presentó niveles significativamente menores comparados con el de la cepa de laboratorio. Las alteraciones observadas en los biomarcadores GST y GSH reflejarían indirectamente la presencia de estrés oxidativo.
\end{abstract}

Palabras clave: carpocapsa, biomarcadores, glutatión S-transferasa, catalasa, superóxido dismutasa, glutatión reducido.

Maero, E. and Anguiano, O. L., 2018. Evaluation of oxidative stress biomarkers in cydia pomonella adults from different populations of Argentine Patagonia. Agriscientia 35: 35-42

\section{SUMMARY}

In a natural environment living organisms are exposed to environmental prooxidant factors. In this study, antioxidant biomarkers were evaluated in adult codling moth Cydia pomonella from different field populations and from 
a laboratory strain. Field populations analyzed were from the provinces of Neuquén (Centenario), Río Negro (Allen, General Roca 1, General Roca 2, General Roca 3 y General Godoy) y Chubut (Sarmiento 1 y Sarmiento 2). The levels of antioxidants were evaluated, for both enzymatic antioxidants such as catalase (CAT), superoxide dismutase (SOD) and glutathione S-transferase (GST) and non-enzymatic, endogenous glutathione (GSH). GST activity found in all field populations were significantly lower than in the laboratory strain. CAT activity in General Roca 1 population turned 2.13 times lower than that from the reference strain. No significant differences in SOD activity were observed between different populations. GSH levels observed in Sarmiento 2 codling moths were significantly different and rise 1.57 times higher than the laboratory ones. Except for General Godoy, all field populations tested had significantly lower GSH levels compared with the laboratory strain. Alterations in GST and GSH biomarkers could indirectly reflect the presence of oxidative stress.

Key words: codling moth, biomarkers, glutathione S-transferase, catalase, superoxide dismutase, reduced glutathione.

Maero, E.: Facultad de Ciencias Agrarias, Universidad Nacional del Comahue. CC: 851 - (8303) Cinco Saltos, Río Negro, Argentina. Anguiano, O. L.: Facultad de Ingeniería, Instituto de Investigación y Desarrollo en Ingeniería de Procesos, Biotecnología y Energías Alternativas, PROBIEN (CONICET-UNCo), Buenos Aires 1400, Neuquén (8300), Argentina. Correspondencia a: elizabeth.maero@faca.uncoma.edu.ar

\section{INTRODUCCIÓN}

Las especies reactivas de oxígeno (ERO), que son o pueden generar fácilmente radicales libres y/o causar daño oxidativo, incluyen a moléculas como el peróxido de hidrógeno $\left(\mathrm{H}_{2} \mathrm{O}_{2}\right)$, aniones como el superóxido $\left(\mathrm{O}_{2}{ }^{--}\right)$y al radical hidroxilo $\left(\mathrm{OH}^{\circ}\right)$ (Halliwell, 2011). Estas especies reactivas, en particular el radical hidroxilo, pueden reaccionar con macromoléculas biológicas y conducir al daño oxidativo de proteínas, lípidos y ADN (Bonekamp, Volkl, Fahimi y Schrader, 2009; Sohal y Orr, 2012). El estrés oxidativo resulta del desequilibrio entre la excesiva formación de ERO y la limitada capacidad del sistema biológico para detoxificarlas rápidamente o para reparar el daño resultante (Halliwell y Whiteman, 2004).

Los insectos, de manera similar a otros organismos aeróbicos, poseen un sofisticado sistema enzimático y no enzimático de defensa antioxidante para contrarrestar y regular el nivel de las ERO. Las enzimas claves para la detoxificación de las ERO son: la superóxido dismutasa (SOD), la catalasa (CAT), la glutatión peroxidasa (GPx) y la glutatión S-transferasa (GST). La SOD cataliza la dismutación del radical superóxido a peróxido y oxígeno. Las enzimas CAT y GPx catalizan la descomposición del peróxido de hidrógeno en agua y oxígeno. Las GST catalizan la conjugación del glutatión reducido (GSH) con una variedad de compuestos electrofílicos que participan en la defensa contra el estrés oxidativo, dado que estas enzimas son capaces de detoxificar sustancias nocivas producto de la peroxidación de lípidos y del ADN entre otras (Ahmad y Pardini, 1990; Felton y Summers, 1995). La defensa antioxidante no enzimática incluye a biomoléculas como el GSH, el ácido ascórbico o vitamina $\mathrm{C}$, el $\alpha$-tocoferol o vitamina $E$, entre otras, que pueden reaccionar con una variedad de radicales libres en los sistemas biológicos eliminando de manera efectiva las especies potencialmente dañinas (Yu, 1994; Felton y Summers, 1995; Birben, Sahiner, Sackesen, Erzurum y Kalaysi, 2012). La alteración de cualquiera de estos parámetros puede reflejar indirectamente la presencia de estrés oxidativo, por lo que pueden actuar como biomarcadores de exposición o de efecto (Peña-Llopis, Ferrando y Peña, 2002; Pandey et al. 2003; Farombi, Adelowo y Ajimoko, 2007; Büyükgüzel, 2009; Birben et al., 2012).

Cydia pomonella (L.) (Lepidoptera: Tortricidae) o carpocapsa es la principal plaga de importancia económica a nivel mundial en cultivos de manzanos, perales y nogales (Reuveny y Cohen, 2004). Durante muchos años, la aplicación de insecticidas fue la herramienta más comúnmente utilizada para el control de esta plaga en la región de la Patagonia Norte, Argentina. A partir del año 2006, la implementación de programas de manejo integrado de plagas (MIP) condujo a la 
reducción significativa del número de aplicaciones de insecticidas neurotóxicos, particularmente organofosforados y carbamatos, los que fueron paulatinamente reemplazados por otros más específicos pertenecientes a nuevos grupos químicos (neonicotinoides y diamidas antranílicas) con distintos modos de acción (Cichón y Garrido, 2012). Si bien, la aplicación de insecticidas para el control de $C$. pomonella se realiza en los estadios tempranos de su ciclo de vida, los insectos adultos también estarían expuestos a estos químicos (Knight y Flexner, 2007). Numerosos trabajos han reportado exposición a plaguicidas que pueden inducir estrés oxidativo al generar radicales libres y alterar el sistema antioxidante en diferentes especies (Ferrari, Venturino y Pechén de D'Angelo, 2007; Kristoff, Verrengia Guerrero y Cochón, 2008; Büyükgüzel, 2009; Dorts et al 2009; Ferreira, Costa da Motta, Kreutz, Toni, Loro y Gil Barcellos, 2010; El-Demerdash, 2011; Akbar, Sharm, Jayalakshmi y Sreeramulu, 2012; JanakiDevi, Nagarani, YokeshBabu, Kumaraguru y Ramakritinan, 2013; Han, Jinhua Wang, Wang, Zhang y Zhu, 2016). Con el objetivo de examinar el sistema antioxidante de C. pomonella, se evaluaron biomarcadores de estrés oxidativo en adultos de ocho poblaciones de campo y de una cepa de laboratorio.

\section{MATERIALES Y MÉTODOS}

\section{Material biológico}

Las determinaciones bioquímicas se realizaron en polillas de $C$. pomonella de uno o dos días de edad. Los insectos adultos se obtuvieron en el laboratorio a partir de larvas diapausantes recolectadas en cartones corrugados colocados en la base de árboles frutales de ocho chacras con manejo convencional, orgánico y sin tratamiento de diferentes localidades patagónicas correspondientes a las provincias de Neuquén, Río Negro y Chubut (Tabla
1), en la temporada 2012-2013. Los adultos de la cepa de referencia se obtuvieron a partir de larvas diapausantes de $C$. pomonella procedentes de una colonia de laboratorio establecida en 1991, criadas en condiciones controladas de temperatura, fotoperíodo, humedad y sin contacto con plaguicidas. Los cartones corrugados con las larvas se colocaron en potes de telgopor de $1 \mathrm{~L}$ cubiertos con tela mosquitera a $25^{\circ} \mathrm{C}$ y fotoperíodo 16:8 (L-O) h hasta la emergencia de los adultos. Todos los insectos emergidos de las diferentes poblaciones evaluadas se recolectaron y guardaron en grupos de a cuatro en tubo de microcentrífuga Eppendorf a $-80^{\circ} \mathrm{C}$ hasta su procesamiento. Los organismos fueron suministrados por el INTA Alto Valle. La designación de las poblaciones corresponde a las localidades de origen de las muestras. Las poblaciones de polillas de diferentes chacras de una misma localidad se identificaron además con un número.

\section{Preparación de los extractos}

Para las determinaciones bioquímicas se utilizaron los abdómenes-tórax de cuatro insectos adultos de C. pomonella que se homogeneizaron en $1.100 \mu \mathrm{L}$ de buffer fosfato de potasio $143 \mathrm{mM} \mathrm{pH}$ 7,5, EDTA 6,3 mM con un homogeneizador Ultrasónico Eléctrico PRO 200, siempre trabajando en hielo. Los homogenados se centrifugaron a 1000 $x \mathrm{~g}$ a $4{ }^{\circ} \mathrm{C}$ durante $15 \mathrm{~min}$. De los sobrenadantes obtenidos se separaron 10 y $150 \mu \mathrm{L}$ para cuantificar proteínas y GSH, respectivamente. El contenido de GSH se determinó inmediatamente. El resto del sobrenadante se centrifugó a 16.000 x g a $4{ }^{\circ} \mathrm{C}$ durante 20 min y el sobrenadante obtenido se separó en alícuotas para determinar las actividades de SOD $(250 \mu \mathrm{L})$, CAT y GST $(200 \mu \mathrm{L})$ y $20 \mu \mathrm{L}$ para cuantificar proteínas. Los sobrenadantes obtenidos se conservaron en ultrafreezer a $-80{ }^{\circ} \mathrm{C}$ hasta su utilización para las determinaciones enzimáticas.

Tabla 1. Descripción de las poblaciones analizadas.

\begin{tabular}{lclc}
\hline Región & \multicolumn{1}{c}{ Localización } & Población & Protección \\
\hline Neuquén & $38^{\circ} 49^{\prime} 00^{\prime \prime} \mathrm{S}, 68^{\circ} 08^{\prime} 00^{\prime \prime} \mathrm{O}$ & Centenario & Química \\
Río Negro & $39^{\circ} 02^{\prime} 45,7^{\prime \prime} \mathrm{S}, 67^{\circ} 43^{\prime} 34,5^{\prime \prime} \mathrm{O}$ & Allen & Química \\
Río Negro & $39^{\circ} 02^{\prime} 31,4^{\prime \prime} \mathrm{S}, 67^{\circ} 33^{\prime} 51,6^{\prime \prime} \mathrm{O}$ & Gral. Roca1 & No tratada* \\
Río Negro & $39^{\circ} 00^{\prime} 36,7^{\prime \prime} \mathrm{S}, 67^{\circ} 41^{\prime} 48,9^{\prime \prime} \mathrm{O}$ & Gral. Roca2 & Química \\
Río Negro & $39^{\circ} 02^{\prime} 12^{\prime \prime} \mathrm{S}, 67^{\circ} 36^{\prime} 56^{\prime \prime} \mathrm{O}$ & Gral. Roca3 & Química \\
Río Negro & $39^{\circ} 05^{\prime} 50,8^{\prime \prime} \mathrm{S}, 67^{\circ} 10^{\prime} 08,7^{\prime \prime} \mathrm{O}$ & Gral. Godoy & Química \\
Chubut & $31^{\circ} 32^{\prime} 15^{\prime \prime} \mathrm{S}, 68^{\circ} 31^{\prime} 11^{\prime \prime} \mathrm{O}$ & Sarmiento1 & Orgánica \\
Chubut & $31^{\circ} 32^{\prime} 15^{\prime \prime} \mathrm{S}, 68^{\circ} 32^{\prime} 25^{\prime \prime} \mathrm{O}$ & Sarmiento2 & Química
\end{tabular}

* Chacra abandonada desde el año 2001 


\section{Análisis bioquímicos}

La actividad GST se determinó usando la técnica desarrollada por Habig, Pabst y Jacoby (1974). La mezcla de reacción consistió en $920 \mu \mathrm{L}$ de bu-

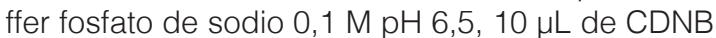
$50 \mathrm{mM}$ (en acetonitrilo) y $50 \mu \mathrm{L}$ de GSH 0,1 mM. Los cambios en la absorbancia fueron registrados continuamente a $340 \mathrm{~nm}$ durante $120 \mathrm{~s}$ a $25^{\circ} \mathrm{C}$ en un espectrofotómetro Shimadzu UV-visible. La actividad específica se expresó como $\mu$ moles de CDNB conjugados $\mathrm{min}^{-1} \mathrm{mg}$ proteína-1 usándose el coeficiente de extinción molar 9,6 $\mathrm{mM}^{-1} \mathrm{~cm}^{-1}$.

La actividad SOD se determinó por su habilidad de inhibir la autooxidación de la epinefrina (Misra y Fridovich, 1972). La mezcla de reacción consis-

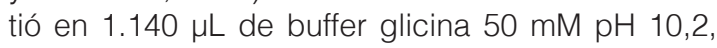
epinefrina $60 \mathrm{mM} \mathrm{pH} 2$ y diferentes volúmenes del sobrenadante (40; 60; 80; 100 y $120 \mu \mathrm{L})$. Se monitoreó el cambio en la absorbancia a $480 \mathrm{~nm}$ durante $600 \mathrm{~s}$ a $30^{\circ} \mathrm{C}$. Los resultados se expresaron como unidades de SOD mg de proteína-1 ( U SOD mg-1). Se define $1 \cup$ de SOD como la cantidad de muestra que causa el $50 \%$ de inhibición de la autooxidación de la epinefrina.

La actividad CAT se determinó según la técnica de Beers y Sizer (1952). La mezcla de reacción consistió en $2990 \mu \mathrm{L}$ de buffer fosfato de sodio 50 $\mathrm{mM}, \mathrm{pH} 7,2{\text { y } \mathrm{H}_{2} \mathrm{O}_{2}}_{2} 5 \mathrm{mM}$. Para iniciar la reacción se agregaron $10 \mu \mathrm{L}$ del extracto enzimático y la actividad CAT se determinó registrando la disminución continua de la absorbancia a $240 \mathrm{~nm}$ durante 60 segundos a $25^{\circ} \mathrm{C}$. La actividad específica se expresó como mmoles de $\mathrm{H}_{2} \mathrm{O}_{2}$ consumidos min ${ }^{-1}$ mg proteína ${ }^{-1}$, utilizando un coeficiente de extinción molar de $40 \mathrm{M}^{-1} \mathrm{~cm}^{-1}$.

El contenido de GSH endógeno se determinó como tioles ácidos solubles mediante la técnica descripta por Ellman (1959) y modificada por Venturino (2001). Se determinó el contenido de GSH en $100 \mu \mathrm{L}$ del sobrenadante al que se le agregó 1 $\mathrm{mL}$ de DTNB 1,5 mM en buffer fosfato de potasio $0,25 \mathrm{M}, \mathrm{pH} 8,0$. La mezcla se incubó durante 20 min a temperatura ambiente y se determinó la absorbancia a $412 \mathrm{~nm}$. Los tioles ácidos solubles se cuantificaron usando una curva de calibración con GSH puro como estándar. Los resultados se expresaron en nmoles de GSH mg de proteína ${ }^{-1}$.

\section{Determinación de proteínas}

La concentración de proteínas se determinó aplicando el método descripto por Lowry, Rosebrough, Farr y Randall (1951) y se usó albúmina sérica bovina como estándar.

\section{Análisis estadísticos}

Los resultados se expresaron como promedios \pm errores estándar de diez muestras independientes. La normalidad de los datos se evaluó mediante el análisis de los residuales y la homocedasticidad a través del test de Levene. Las diferencias estadísticas de los parámetros bioquímicos evaluados entre las poblaciones se analizaron por ANOVA de un factor seguido del test de Tukey, usando el software Statistica 7.1. Los datos de GST se transformaron con raíz cuadrada para lograr la normalidad de las varianzas. El nivel de significancia $p<0,05$ se utilizó en todos los análisis estadísticos.

\section{RESULTADOSY DISCUSIÓN}

En los últimos años, se ha intensificado notablemente el interés en el estudio de las enzimas GST en artrópodos por su rol potencial en el mantenimiento del estado redox celular (Ranson y Hemingway, 2005; Li, Schuller y Berenbaum, 2007; Fang, 2012). La actividad GST determinada en insectos adultos de $C$. pomonella de diferentes poblaciones se presenta en la Figura 1. Los niveles de actividad observados en todas las poblaciones de campo resultaron significativamente diferentes, entre 1,5 y 3,3 veces menores al detectado en las polillas de laboratorio. También, Frenzilli et al. (2004), detectaron una disminución significativa de la actividad GST en moluscos Mytilus galloprovincialis después de siete días de trasplantados a sitios contaminados en el puerto de Génova. De Luca-

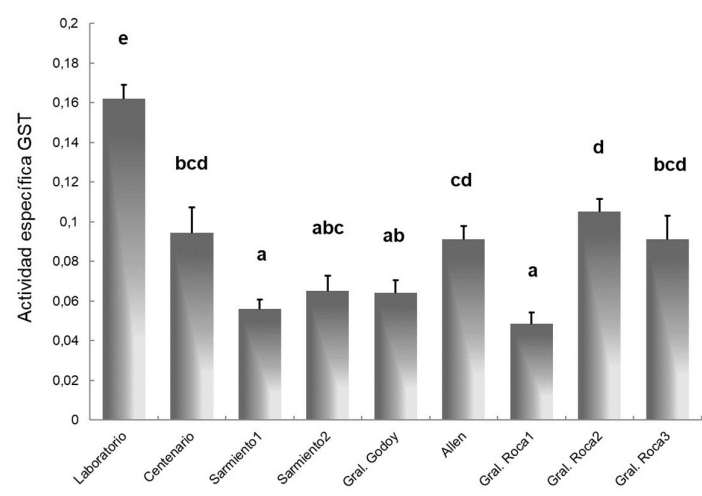

Figura 1. Actividad de GST en adultos de C. pomonella de ocho chacras de manzanos de la Patagonia argentina y de una cepa de laboratorio. Cada uno de los puntos representa la actividad promedio \pm el error estándar de diez muestras independientes. La actividad está expresada como $\mu$ moles de CDNB conjugado $\mathrm{min}^{-1} \mathrm{mg}$ proteina ${ }^{-1}$. Valores similares se representan con la misma letra. Letras distintas indican diferencias significativas entre poblaciones, $p<0,05$. 
Abbott et al. (2005) evaluaron la actividad GST en hepatopáncreas de mejillones Perna viridis trasplantados a cuatro sitios sospechados de contaminación química y observaron una disminución significativa de la GST a los 28 días de exposición. La actividad GST disminuida evidenciaría su rol protector reducido y dejaría a la célula expuesta a los efectos deletéreos de las ERO producidas durante el estrés oxidativo. La disminución de la actividad GST podría ser explicada por dos hipótesis diferentes: Según Egaas et al (1999), en la primera etapa de la biotransformación de xenobióticos catalizados por las enzimas citocromo P450 se produciría un cóctel de diferentes metabolitos, los cuales competirían con los sustratos de las GST por su sitio activo. De acuerdo con Gallagher y Sheehy (2000), la menor actividad enzimática podría deberse a la inhibición de la síntesis de las GST.

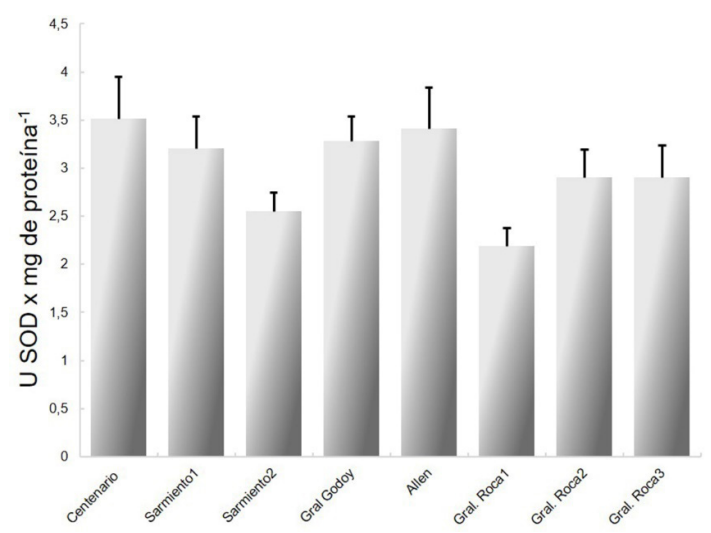

Figura 2. Actividad de SOD en adultos de $C$. pomonella de diferentes poblaciones de campo. Una unidad de SOD (U SOD) se define como la cantidad de muestra que causa el $50 \%$ de inhibición de la autooxidación de la epinefrina. Los valores representan la actividad promedio \pm el error estándar de diez muestras independientes.

Por otra parte, no se observaron diferencias significativas entre las actividades SOD determinadas en adultos de diferentes poblaciones de C. pomonella cuyos valores promedio fluctuaron entre $2,18 \mathrm{y}$ 3,51 U SOD mg de proteína-1 $(p>0,05)$ (Figura 2). Las enzimas SOD y CAT constituyen la primera línea de defensa antioxidante contra las ERO en los organismos aeróbicos. Felton y Summers (1995) detectaron en insectos que la enzima SOD está presente en todos los tipos celulares y es uno de los antioxidantes enzimáticos más efectivos contra el estrés oxidativo. El papel clave de esta enzima en la protección contra los tóxicos prooxidantes se demostró cuando su inhibición resultó en un incremento del efecto tóxico de las ERO (Ahmad y Pardini, 1990).

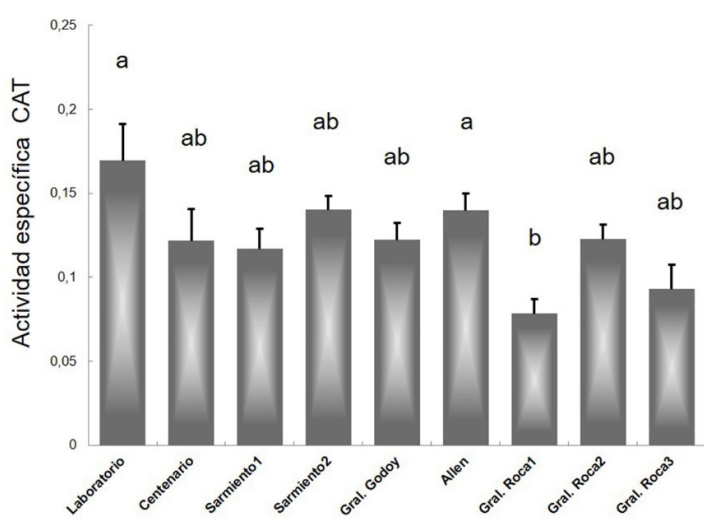

Figura 3. Actividad de CAT en adultos de C. pomonella determinada en ocho poblaciones de campo y en una de laboratorio. Los valores representan la actividad promedio \pm el error estándar de diez muestras independientes. La actividad específica se expresa como mmoles de $\mathrm{H}_{2} \mathrm{O}_{2}$ consumido $\mathrm{min}^{-1} \mathrm{mg}_{\text {proteina }}{ }^{-1}$. Letras distintas indican diferencias significativas entre poblaciones, $p<0,05$.

Nuestros resultados mostraron que la actividad CAT determinada en las polillas de laboratorio fue similar a la de la población de Allen y resultó significativamente superior a la expresada en los individuos provenientes de General Roca 1 $(p<0,01)$ (Figura 3). El resto de las poblaciones ensayadas presentaron actividades menores, aunque no resultaron estadísticamente diferentes. También Pampanin et al (2005) han reportado una disminución significativa de la actividad CAT en mejillones Mytilus galloprovincialis trasplantados a sitios contaminados en el Lago Venecia, Italia. Resultados similares fueron informados por VioqueFernández, Alves de Almeida y López-Barea (2009) en cangrejos Procambarus clarkii recolectados de sitios contaminados cercanos a cultivos de arroz en el sur de España. La enzima CAT facilita la remoción del $\mathrm{H}_{2} \mathrm{O}_{2}$, que es metabolizado a oxígeno molecular y agua. La disminución de la actividad CAT resultaría en la detoxificación deficiente del $\mathrm{H}_{2} \mathrm{O}_{2}$ e indicaría la presencia de químicos inductores de estrés oxidativo. Se conoce que esta enzima es sensible al daño causado por ERO y puede ser inhibida por el radical superóxido (Kono y Fridovich, 1982).

Por otra parte, el nivel medio de GSH determinado en adultos de las poblaciones de laboratorio y de General Godoy fue similar $(22,17 \pm 0,48$ nmoles GSH mg proteína ${ }^{-1}$ y 20,64 $\pm 1,14$ nmoles GSH mg proteína $^{-1}$, respectivamente) (Figura 4). El mayor valor de GSH se observó en las polillas provenientes de Sarmiento 2, que resultó significativamente diferente $(p=0,000135)$ del determinado en el resto de las poblaciones y fue 1,57 veces mayor al obtenido en la cepa de laboratorio. De manera similar, Pandey et al. (2003) detectaron altos niveles de GSH en el pez de agua dulce Wallago 


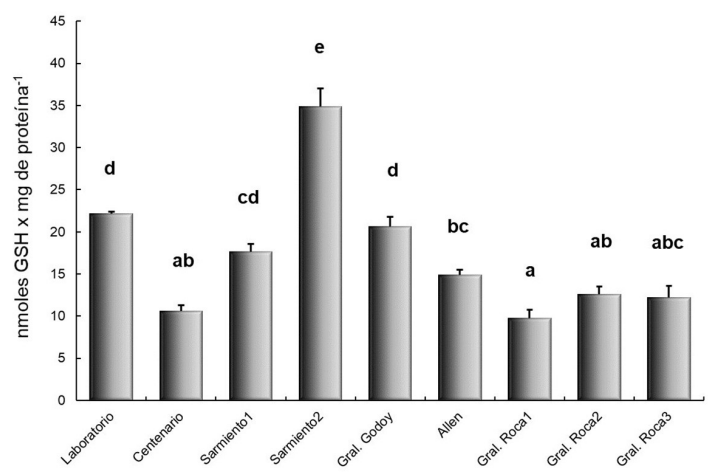

Figura 4. Niveles de GSH endógeno en adultos de C. pomonella obtenidos en ocho poblaciones de campo y en la cepa de laboratorio. Los valores representan el nivel promedio \pm el error estándar de diez muestras independientes. Valores similares se representan con la misma letra. Letras distintas indican diferencias significativas entre poblaciones, $p<0,05$.

attu, proveniente de zonas contaminadas de la India; De Luca-Abbott et al. (2005) en branquias de almejas y mejillones trasplantados a sitios contaminados en la costa de Hong Kong, luego de 14 y 28 días de exposición; y Farombi et al. (2007) en hígado, riñón y corazón del pez gato, Clarias gariepinus, proveniente de un río contaminado de Nigeria. El aumento en el contenido de GSH podría sugerir un papel protector y adaptativo de esta biomolécula contra el estrés oxidativo inducido por compuestos prooxidantes. Además, su incremento se ha relacionado en diversos organismos con el desarrollo de la resistencia a plaguicidas (Peña et al, 2000; Peña-Llopis, Peña, Sancho, FernándezVega y Ferrando, 2001).

Por el contrario, el resto de los individuos estudiados presentó niveles de GSH significativamente menores. En particular, el valor promedio más bajo se determinó en las polillas de General Roca 1 y resultó 2,3 veces menor al de los adultos de laboratorio. También, Farombi et al. (2007) detectaron una disminución significativa del nivel de GSH en branquias del pez gato, Clarias gariepinus, proveniente de un río contaminado de Nigeria. La disminución en el contenido de este antioxidante podría deberse a su utilización como sustrato de enzimas detoxificantes y/o antioxidantes (GST, GPx) y/o a su oxidación directa al reaccionar con ERO, en una situación de estrés oxidativo. Jokanovic (2001) propuso que una disminución igual o mayor al $20 \%$ en el contenido de esta biomolécula resultaría altamente perjudicial para la supervivencia de la célula.

\section{CONCLUSIONES}

Este es el primer estudio que evalúa los niveles basales de biomarcadores de estrés oxidativo en adultos de $C$. pomonella provenientes de diversas localidades de la Patagonia argentina. Los resultados presentados en esta investigación muestran que la actividad GST fue significativamente menor en los individuos de C. pomonella provenientes de ocho chacras con cultivos de manzanos, en comparación con la de la cepa de laboratorio, que sugiere el papel protector disminuido frente a xenobióticos tóxicos. El aumento significativo en el contenido de GSH determinado en las polillas de C. pomonella de Sarmiento 2 indica una respuesta adaptativa de estos organismos a la exposición a prooxidantes. Además, el nivel de esta biomolécula significativamente menor respecto al de la cepa de laboratorio, podría sugerir su oxidación por ERO en situación de estrés oxidativo.

Las alteraciones observadas en los biomarcadores antioxidantes GST y GSH en polillas de $C$. pomonella de las distintas poblaciones de campo sugerirían la exposición a contaminantes y/o factores ambientales prooxidantes. Estudios posteriores serán necesarios para evaluar los efectos adversos del estrés oxidativo en adultos de C. pomonella sobre la repuesta fisiológica en la supervivencia, el crecimiento y la reproducción.

\section{AGRADECIMIENTOS}

Los autores agradecen a la Dra. Liliana Cichón y al personal de campo del INTA Alto Valle por la provisión de los organismos utilizados en este trabajo. El apoyo financiero para esta investigación fue proporcionado por el Proyecto 04/1004 de la Universidad Nacional del Comahue.

\section{BIBLIOGRAFÍA}

Ahmad S. y Pardini, R. S. (1990). Mechanisms for regulating oxygen toxicity in phytophagous insects. Free Radical Biology and Medicine, 8 (4), 401-413.

Akbar, S. M., Sharm, H. C., Jayalakshmi, S. K. y Sreeramulu, K. (2012). Methylparathion- and carbofuran-induced mitochondrial dysfunction and oxidative stress in Helicoverpa armigera (Noctuidae: Lepidoptera). Pesticide Biochemistry and Physiology, 103, 31-37.

Beers, R. F. y Sizer, J. W. (1952). A spectrophotometric method for measuring the breakdown of hydrogen peroxide by catalase. Journal of Biological Chemical, 195 (1), 133-140. 
Birben, E., Sahiner, U. M., Sackesen, C., Erzurum, S. y Kalaysi, O. (2012). Oxidative stress and antioxidant defense. WAO Journal, 5, 9-19.

Bonekamp, N. A., Volkl, A., Fahimi, D. y Schrader, M. (2009). Reactive oxygen species and peroxisomes: struggling for balance. BioFactors, 35 (4). 346-355.

Büyükgüzel, E. (2009). Evidence of oxidative and antioxidative responses by Galleria mellonella larvae to malathion. Journal of Economic Entomology, 102 (1), 152-159.

Cichón, L. y Garrido, S. (2012). Implicancia de los cambios en el manejo sanitario de frutales de pepita en el Alto Valle. Revista Fruticultura \& Diversificación, 67, 8-15.

De Luca-Abbott, S. B., Richardson, B., McClellan, K., Zheng, G. J., Martin, M. y Lam, P. (2005). Field validation of antioxidant enzyme biomarkers in mussels (Perna viridis) and clams (Ruditapes philippinarum) transplanted in Hong Kong coastal waters. Marine PoIlution Bulletin, 51, 694-707.

Dorts, J., Silvestre, F., Thi Tu, H., Tyberghein, A. E., Phuong, N. T. y Kestemont, K. (2009). Oxidative stress, protein carbonylation and heat shock proteins in the black tiges shrimp, Penaeus manodon, following exposure to endosulfan and deltamethrin. Environmental Toxicology and Pharmacology, 28, 302-310.

Egaas, E., Sandvik, M., Fjeld, E., Kallqvist, T., Goksoyr, A. y Svenson, A. (1999). Some effects of the fungicide propiconazole on cytochrome P450 and glutathione Stransferase in brown trout (Salmo trutta). Comparative Biochemistry and Physiology, 122, 337-344.

El-Demerdash, F. M. (2011). Lipid peroxidation, oxidative stress and acetylcholinesterase in rat brain exposed to organophosphate and pyrethroid insecticides. Food and Chemical Toxicology, 49, 1346-1352.

Ellman, G. L. (1959). Tissue sulfhydryl groups. Archives of Biochemistry and Biophysics, 82 (1), 70-77.

Fang, S. M. (2012). Insect glutathione S-transferase: a review of comparative genomic studies and response to xenobiotics. Bulletin of Insectology, 65 (2), 265-271.

Farombi, E. O.; Adelowo, O. A. y Ajimoko, Y. R. (2007). Biomarkers of Oxidative Stress and Heavy Metal Levels as Indicators of Environmental Pollution in African Cat Fish (Clarias gariepinus) from Nigeria Ogun River. International Journal of Environmental Research and Public Health, 4 (2), 158-165.

Felton, G. W. y Summers, C. B. (1995). Antioxidant systems in insects. Archives of Insect Biochemistry and Physiology, 29, 187-197.

Ferrari, A., Venturino, A. y Pechén de D’Angelo, A. M. (2007). Effects of carbaryl and azinphos-methyl on juvenile rainbow trout (Oncorhynchus mykiss) detoxifying enzymes. Pesticide Biochemistry and
Physiology, 88, 134-142.

Ferreira, D., Costa da Motta, A., Kreutz, L. C., Toni, C., Loro, V. L. y Gil Barcellos, L. J. (2010). Assessment of oxidative stress in Rhamdia quelen exposed to agrochemicals. Chemosphere, 79, 914-921.

Frenzilli, G., Bocchetti, M., Nigro, M., Annarumma, F., Scarcelli, V., Fattorini, D. y Regoli, F. (2004). Timecourse evaluation of ROS-mediated toxicity in mussels, Mytilus galloprovincialis, during a field translocation experiment. Marine Environmental Research, 58, 609-613.

Gallagher, E. P. y Sheehy, K. M. (2000). Altered glutathione S-transferase catalytic activities in female brown bullheads from a contaminated central Florida lake. Marine Environmental Research, 50, 399-403.

Habig, W. H., Pabst, M. J. y Jacoby, W. B. (1974). Glutathione S-transferases: The first enzymatic step in mercapturic acid formation. Journal of Biological Chemical, 249, 7130-7139.

Halliwell, B. y Whiteman, M. (2004). Measuring reactive species and oxidative damage in vivo and in cell culture: how should you do it and what do the results mean? British Journal of Pharmacology, 142, 231-255.

Halliwell, B. (2011). Free radicals and antioxidants - quo vadis? Trends in Pharmacological Sciences, 32 (3), 125-130.

Han, Y., Jinhua Wang, T. L., Wang, J., Zhang, C., Zhu, L. (2016). Genotoxicity and oxidative stress induced by the fungicide azoxystrobin in zebrafish (Danio rerio) livers. Pesticide Biochemistry and Physiology, 133, 13-19.

JanakiDevi, V., Nagarani, N., YokeshBabu, M., Kumaraguru, A. K. y Ramakritinan, C. M. (2013). A study of proteotoxicity and genotoxicity induced by the pesticide and fungicide on marine invertebrate (Donax faba). Chemosphere, 90, 1158-1166.

Jokanovi, M. (2001). Biotransformation of organophosphorus compounds. Toxicology, 166 (3), 139-160.

Knight, A. y Flexner, L. (2007). Disruption of mating in codling moth (Lepidoptera: Tortricidae) by chlorantraniliprole, an anthranilic diamide insecticide. Pest Management Science, 63, 180-189.

Kono, Y. y Fridovich, I. (1982). Superoxide Radical Inhibits Catalase. The Journal of Biological Chemistry, 257 (10), 5751-5754

Kristoff, G., Verrengia Guerrero, N. R. y Cochón, A. C. (2008). Effects of azinphos-methyl exposure on enzymatic and non-enzymatic antioxidant defenses in Biomphalaria glabrata and Lumbriculus variegatus. Chemosphere, 72 (9), 1333-1339.

Li, X., Schuller, M. A. y Berenbaum, M. R. (2007). Molecular mechanisms of metabolic resistance to synthetic and natural xenobiotics. Annual Review of Entomology, 52, 231-253. 
Lowry, O. H., Rosebrough, N. J., Farr, A. L. y Randall, R. J. (1951). Protein measurement with the Folin phenol reagent. The Journal of Biological Chemistry, 193, 265-275.

Misra, H. P. y Fridovich, I. (1972). The role of superoxide anion in the autoxidation of epinephrine and a simple assay for superoxide dismutase. Journal of Biological Chemistry, 247 (10), 3170-3175.

Pampanin, D. M., Camus, L., Gomiero, A., Marangon, I., Volpato, E. y Nasci, C. (2005). Susceptibility to oxidative stress of mussels (Mytilus galloprovincialis) in the Venice Lagoon (Italy). Marine Pollution Bulletin, 50, 1548-1557.

Pandey, S., Parvez, S., Sayeed, I., Haque, R., Bin-Hafeez, B. y Raisuddin, S. (2003). Biomarkers of oxidative stress: a comparative study of river Yamuna fish Wallagu attu (BI. and Schn.). The Science of the Total Environment, 309, 105-115.

Peña, S., Peña, J. B., Ríos, C., Sancho, E., Fernández, C. y Ferrando, M. D. (2000). Role of glutathione in thiobencarb resistance in the European eel Anguilla anguilla. Ecotoxicology and Environmental Safety, 46 (1), 51-56.

Peña-Llopis, S., Peña, J. B., Sancho, E., Fernández-Vega, C. y Ferrando, M. D. (2001). Glutathione-dependent resistance of the European eel Anguilla anguilla to the herbicide molinate. Chemosphere, 45 (1), 671-681.
Peña-Llopis, S., Ferrando, M. D. y Peña, J. B. (2002). Impaired glutathione redox status is associated with decreased survival in two organophosphate-poisoned marine bivalves. Chemosphere 47, 485-497.

Ranson, H. y Hemingway, J. (2005). Mosquito glutathione transferases. Methods in Enzymology, 401, 226-241.

Reuveny, H. y Cohen, E. (2004). Evaluation of mechanisms of azinphos-methyl resistance in the codling moth Cydia pomonella (L.). Archives of Insect Biochemistry and Physiology, 57 (2), 92-100.

Sohal, R. S. y Orr, W. C. (2012). The redox stress hypothesis of aging. Free Radical Biology and Medicine, 52, 539-555.

Venturino, A., Anguiano, O. L., Gauna, L., Cocca, C., Bergoc, R. M. y Pechen de D'Angelo, A. M. (2001). Thiols and polyamines in the potentiation of malathion toxicity in larval stages of the toad Bufo arenarum. Comparative Biochemistry and Physiology, 130C, 191-198.

Vioque-Fernández, A., Alves de Almeida, E. A. y LópezBarea, J. (2009). Assessment of Doñana National Park contamination in Procambarus clarkii: Integration of conventional biomarkers and proteomic approaches. Science of the total Environment, 407 (5), 1784-179.

Yu, B. P. (1994). Cellular defences against damage from reactive oxygen species. Physiological Reviews, 74, 139-162. 